\title{
ESTUDO DE HOMOGENEIDADE DE LOTE DE MATERIAL SILÍCIO METÁLICO CANDIDATO A MATERIAL DE REFERENCIA
}

\author{
Queenie Siu Hang Chui*
}

Unidade Acadêmica das Áreas de Ciências Exatas e Tecnológicas, Universidade São Francisco, Rua Alexandre Rodrigues Barbosa, 45, 13251-900 Itatiba - SP

Celia Omine Iamashita e João Marcos de Almeida Bispo

Divisão de Química, Instituto de Pesquisas Tecnológicas do Estado de São Paulo S.A., Av. Prof. Almeida Prado, 535, 05508-901 São Paulo - SP

Recebido em 17/5/04; aceito em 22/10/04; publicado na web em 17/2/05

\begin{abstract}
HOMOGENEITY TESTING OF A LOT OF SILICON METAL PREPARED AS A REFERENCE MATERIAL. From the perspective of the uncertainties in chemical measurements all uncertainty sources should be part of the uncertainty of the reference material. When the primary methods are not available, interlaboratorial comparisons are used as a means of certification. The material to be distributed to the laboratories should have its homogeneity confirmed. The uncertainty due to this factor will be added to the characterization uncertainty. This work presents a homogeneity study of a lot of silicon metal of chemical degree where the uncertainty due to inhomogeneity is obtained using analysis of variance.
\end{abstract}

Keywords: uncertainty; homogeneity test; reference material.

\section{INTRODUÇÃO}

Nas últimas décadas, a queda das barreiras protecionistas permitiu o crescimento do comércio internacional, reforçado pelas demandas devido às exigências do mundo globalizado. A realização das trocas de mercadorias, através dos valores verdadeiros dos produtos, representa um dos aspectos que contribui para o crescimento econômico de um país e demonstra a importância de se ter resultados de análises e ensaios químicos com confiabilidade metrológica.

$\mathrm{O}$ resultado de uma medição tem como principal objetivo dar suporte à tomada de decisões. Em situações de inspeção e/ou perícia, os resultados devem ser comparáveis aos obtidos em outros laboratórios. Nessa condição, os resultados dos diversos laboratórios devem estar relacionados à mesma referência.

A inserção das questões da Metrologia, ciência que se dedica ao aprimoramento de medições em todas as áreas do conhecimento, tem impulsionado o química analista a se defrontar com o estabelecimento da rastreabilidade ${ }^{1}$ às medições geradas em seus experimentos e ensaios.

O significado da palavra "rastreabilidade" em medições é explicada nos termos do $\mathrm{VIM}^{2}$ (Vocabulário Internacional de Metrologia). No sentido genérico, diz respeito à "propriedade do resultado de uma medição estar relacionado a referências estabelecidas, geralmente padrões nacionais e internacionais, através de uma cadeia contínua de comparações, todas tendo as incertezas estabelecidas". O conceito é geralmente expresso pelo adjetivo "rastreável" e a cadeia contínua de comparações é denominada "cadeia de rastreabilidade".

Mas como obter resultados analíticos rastreáveis e comparáveis $^{3}$ aos obtidos por outros laboratórios dispersos no mundo todo?

Como observado acima, padrões são freqüentemente utilizados como referências na cadeia de rastreabilidade, em todos os setores da Metrologia, seja em medições físicas, mecânicas, químicas, bioquímicas e biológicas, entre outros ${ }^{4}$.

*e-mail: queenie.hang@saofrancisco.edu.br
Para a Química, pode-se dizer que existem os padrões químicos (como ex. o isótopo Carbono 12, relacionado ao número de Avogadro e o Faraday) que dão rastreabilidade aos padrões analíticos, primários ou secundários, e que representam a ligação ao sistema SI.

No dia-a-dia de um laboratório de análises químicas são necessários padrões cujas propriedades apresentam valores próximos aos esperados e que se relacionem com as propriedades de interesse, em diferentes matrizes reais. Dentro dessa classe ${ }^{5}$ encontramse os Materiais de Referência (MRs), que são "substâncias que têm um ou mais valores de propriedades suficientemente homogêneos e bem estabelecidos para serem usados na calibração de um aparelho, na avaliação de um método de medição ou atribuição a valores ou materiais" e os Materiais de Referência Certificados (MRCs), que são "materiais de referência com valores certificados para uma ou mais propriedades de interesse".

Nos últimos anos, observa-se uma ênfase maior para a utilização de materiais de referência como forma de validação ${ }^{6}$ de medições em química analítica. A qualidade dos materiais de referência tem influência direta sobre a qualidade dos resultados analíticos gerados em laboratórios ${ }^{7}$. Em geral, os materiais são certificados por procedimentos especiais, sob supervisão de um órgão independente e competente. Quando não estão disponíveis métodos primários $^{1}$, são utilizados programas interlaboratoriais especialmente planejados para esse fim ${ }^{8}$. Os MRCs são conhecidos também como padrões de matrizes reais, pois, regra geral, são preparados a partir de materiais naturais processados, de modo a simular as condições presentes em amostras naturais, submetidos a processos controlados de medição (ex. sedimentos de rios com composição química certificada, impurezas presentes em produtos metalúrgicos, etc.)

A certificação de materiais de referência pressupõe oferecer ao usuário valores atribuídos às propriedades de interesse, que servirão de meios para conferir a rastreabilidade a medições geradas em processos metrológicos ${ }^{4}$, que fazem parte dos trabalhos de pesquisa e desenvolvimento, de controle de qualidade e de outros fins.

As quatro etapas que compõem os trabalhos para obtenção de materiais de referência sólidos consistem em preparar o material 
na granulometria desejada, distribuí-lo em frascos e verificar se a homogeneidade do material nos frascos é confirmada, fazer o teste para estabelecer o tempo de estabilidade que poderá ser garantido ao material embalado e a ser mantido em estoque, e a certificação dos valores a serem atribuídos às propriedades de interesse do material preparado.

A abordagem das incertezas em medições químicas, conforme preconizada pelos assuntos da Metrologia indicada na norma ISO $35^{8}$, que dispõe sobre os procedimentos para a certificação de materiais de referência, pressupõe a declaração das incertezas de todas as etapas de sua preparação; é exigido do produtor de materiais de referência agregar à incerteza dos valores certificados a parcela devida à caracterização e, mais recentemente, a parcela devida à homogeneidade ${ }^{3,7}$ do material que compõe o lote preparado e que representa as amostras embaladas em frascos, cilindros (se os materiais certificados forem gases) ou outras formas de embalagem; e a parcela devida à estabilidade, ou seja, a possíveis efeitos de degradação do material de referência após seu preparo.

A incerteza padrão combinada ${ }^{9}$ para um MRC $\left(\mathrm{u}_{\mathrm{MRC}}\right)$ pode ser expressa pela Equação 1:

$\mathrm{u}_{\mathrm{MRC}}=\left(\mathrm{u}_{\text {caracterização }}^{2}+\mathrm{u}_{\text {homogeneidade }}^{2}+\mathrm{u}_{\text {estabilidade }}^{2}\right)^{1 / 2}$

Nesta equação, $\mathrm{u}^{2}$ homogeneidade é obtida em experimento planejado para responder a seguinte questão: será que todos os frascos ou outras formas de embalagem disponibilizadas contêm amostras de MRC suficientemente homogêneas? Se houver diferenças entre frascos, quanto dessas diferenças representam a incerteza total do valor certificado?

$\mathrm{Na}$ Equação 1, $\mathrm{u}^{2}{ }_{\text {estabilidade }}$ expressa a parcela devida à possível degradação ${ }^{7,10}$ do material certificado e deve ser considerada para a composição da incerteza final associada ao valor da propriedade certificada. É indicado o monitoramento ao longo do tempo, por pelo menos $2 \operatorname{anos}^{10}$, para verificar se existem efeitos devido à estocagem em diferentes temperaturas ou às condições de transporte a que os materiais podem ser expostos. Na prática, observase que minérios e materiais metálicos muito raramente sofrem decomposição devido à temperatura; porém, é recomendada sua estocagem em frascos hermeticamente fechados e mantidos em ambientes climatizados. Em geral, a validade de um MRC é estabelecida como garantia de estocagem. Cabe ao usuário adotar práticas que assegurem a qualidade do produto para sua utilização após aberta a embalagem. Em laboratórios, é recomendada a manutenção de gráficos de controle para o acompanhamento da estabilidade do produto até seu esgotamento; dessa forma demonstrando-se a confiabilidade de seus resultados e de modo a prover-se de evidências técnicas, quando solicitados para o atendimento dos requisitos técnicos para a garantia da qualidade analítica ${ }^{11}$.

Esse trabalho apresenta o estudo de homogeneidade de um lote de material de silício metálico grau químico candidato a MRC e, como consequiência, obter a incerteza devido às características de homogeneidade do lote preparado.

O planejamento do experimento para verificar a homogeneidade de um lote de material preparado para fins de certificação deve indicar as variabilidades devido a amostras dentro de frascos e as variabilidades de amostras entre os frascos ${ }^{12,13}$, que contêm os materiais que compõem o lote, devidamente envasados. A questão comum a ser estudada é distinguir os efeitos de heterogeneidade do material dos efeitos devido às variabilidades intrínsecas às próprias medições.

\section{PREPARAÇÃO DO MATERIAL}

O material bruto silício metálico contendo $98,5 \%$ Si foi fornecido pela empresa LIASA - Ligas de Alumínio S.A., localizada no Distrito Industrial do município de Pirapora, estado de Minas Gerais.

Uma quantidade de aproximadamente $60 \mathrm{~kg}$ do material bruto recebido foi preparada em moinho de panela recoberto de carbeto de tungstênio e, após peneirada, foi separada a fração retida entre as peneiras de abertura de malha para conter partículas entre 60 a $75 \mu \mathrm{m}$.

$\mathrm{O}$ material resultante da moagem e peneiramento, aproximadamente $32 \mathrm{~kg}$, foi homogeneizado pelo método de quarteamento manual, usando filme plástico, após o que foi disposto em quatro baldes de polietileno de $25 \mathrm{~L}$.

O material do balde 1 foi distribuído em frascos identificados como de $\mathrm{n}^{\text {os }} 1$ a 149 ; do balde 2 , de $\mathrm{n}^{\text {os }} 150$ a 296 ; do balde 3 , de $\mathrm{n}^{\text {os }}$ 297 a 442 e do balde 4, de $n^{\text {os }} 443$ a 475 . Os frascos eram de vidro incolor com tampas pretas de plástico e receberam etiquetas adesivas para a devida identificação. Cada frasco, contendo $60 \mathrm{~g}$ de material, foi identificado como IPT 134, numerados de 1 a 475.

Cinco frascos provenientes de cada um dos 4 baldes, totalizando 20 frascos, foram selecionados aleatoriamente e encaminhados para os ensaios químicos no Laboratório de Materiais de Referência Inorgânicos da Divisão de Química do IPT, que usou a técnica da Espectrometria de Emissão por Plasma de Argônio Induzido (ICPOES), para determinar $\mathrm{Fe}, \mathrm{Ca}, \mathrm{Al}$, Ti e $\mathrm{Mn}$.

\section{RESULTADOS E DISCUSSÃO}

A avaliação estatística dos resultados foi obtida usando a análise de variâncias fator único (baldes). A Tabela 1 mostra todos os resultados obtidos para a determinação dos elementos $\mathrm{Fe}, \mathrm{Ca}, \mathrm{Al}$, Mn e Ti.

Tabela 1. Resultados da determinação de Fe, Ca, Al, Mn e Ti (em \%)

\begin{tabular}{lccccc}
\hline frasco & $\mathrm{Fe}$ & $\mathrm{Ca}$ & $\mathrm{Al}$ & $\mathrm{Mn}$ & $\mathrm{Ti}$ \\
\hline 1 & 0,283 & 0,104 & 0,083 & 0,0120 & 0,0103 \\
24 & 0,275 & 0,106 & 0,090 & 0,0120 & 0,0104 \\
47 & 0,281 & 0,112 & 0,089 & 0,0113 & 0,0109 \\
70 & 0,281 & 0,109 & 0,088 & 0,0115 & 0,0109 \\
93 & 0,280 & 0,108 & 0,088 & 0,0116 & 0,0106 \\
150 & 0,282 & 0,110 & 0,090 & 0,0117 & 0,0110 \\
173 & 0,274 & 0,109 & 0,090 & 0,0120 & 0,0106 \\
196 & 0,280 & 0,108 & 0,092 & 0,0117 & 0,0106 \\
219 & 0,285 & 0,105 & 0,084 & 0,0120 & 0,0102 \\
242 & 0,280 & 0,109 & 0,085 & 0,0120 & 0,0104 \\
297 & 0,275 & 0,111 & 0,089 & 0,0120 & 0,1080 \\
320 & 0,282 & 0,104 & 0,087 & 0,0121 & 0,0104 \\
343 & 0,282 & 0,107 & 0,088 & 0,0117 & 0,0109 \\
366 & 0,282 & 0,109 & 0,089 & 0,0116 & 0,0109 \\
389 & 0,277 & 0,108 & 0,089 & 0,0119 & 0,0105 \\
443 & 0,282 & 0,109 & 0,088 & 0,0121 & 0,0105 \\
449 & 0,286 & 0,106 & 0,089 & 0,0120 & 0,0106 \\
455 & 0,280 & 0,110 & 0,084 & 0,0115 & 0,0108 \\
461 & 0,275 & 0,109 & 0,088 & 0,0117 & 0,0105 \\
467 & 0,285 & 0,108 & 0,088 & 0,0115 & 0,0110 \\
\hline
\end{tabular}

Os resultados da análise de variâncias para o caso de ferro $(\mathrm{Fe})$ estão mostrados na Tabela 2. As 5 determinações em cada balde, denominados de 1 a 4, correspondem aos frascos numerados e mostrados na Tabela 1 e podem ser identificados na sua respectiva coluna. As informações da Tabela 2 apresentam as médias obtidas para as repetições em cada balde e a decomposição das variâncias extrínsecas e intrínsecas aos baldes. 
Através da decomposição das variâncias, observa-se que o teste $\mathrm{F}_{(\alpha=0,05: 3,16)}$ indicou o valor $\mathrm{F}_{\text {calculado }}$ de 0,2712 , bem menor que o $\mathrm{F}_{\text {crítico }}$ de 3,2389 , não demonstrando motivo para não aceitação de homogeneidade entre os baldes.

A Tabela 3 indica os resultados para médias e variâncias dos resultados de cálcio $(\mathrm{Ca})$ em cada balde e a decomposição das variâncias.

Para cálcio, $\mathrm{F}_{\text {calculado }}(0,08219)<\mathrm{F}_{\text {crítico }}(3,2389)$ indica que não há diferenças entre as médias.

Para os demais elementos, Al, Mn e Ti, os resultados estão mostrados nas Tabelas 4,5 e 6 .

Para todos os elementos citados nas Tabelas 4 a 6 , o teste $\mathrm{F}$ confirmou não haver diferenças significativas, com $\mathrm{F}_{\text {calculado }}<\mathrm{F}_{\text {crítico }}$.

Comparando os valores de $\mathrm{F}_{\text {calculado }}$ para os elementos estudados de 0,1881 para Al; de 0,7048 para Mn e de 0,2996 para Ti , com o valor de $\mathrm{F}_{\text {crítico }}=3,2389$, pôde-se dizer que não houve diferenças significativas entre as médias obtidas nos 4 baldes.

Considerando que as médias quadráticas entre grupos e dentre grupos representam as variâncias extrínseca e intrínseca entre baldes, pode-se calcular a variância entre baldes $\left(\mathrm{s}^{2}{ }_{\text {entre baldes }}\right)$, usando a Equação 2:

$s_{\text {entre baldes }}^{2}=\frac{\left(M Q_{\text {entregrupos }}\right)-\left(M Q_{\text {dentregrupos }}\right)}{n}$

sendo $n=\mathrm{n}^{\circ}$ de replicatas efetuadas em cada balde.

Com os resultados obtidos nos casos estudados, ao calcular a parcela referente à variância entre baldes, obtiveram-se valores negativos, da ordem de $10^{-9}$ que, na prática, são interpretados como nulos $^{12,13}$. Nessa situação, é recomendado considerar a contribuição da variância dentre grupos, ou seja, da repetitividade das replicatas. Sendo assim, a incerteza padrão devido à não homogeneidade $\left(\mathrm{u}_{\text {homogeneidade }}\right)$ do lote preparado será representada pela Equação $3^{12}$ :

Tabela 2. Resultados da análise de variâncias - Determinação de ferro

\begin{tabular}{ccccc}
\hline$\%$ Fe & balde 1 & balde 2 & balde 3 & balde 4 \\
\hline 1 & 0,283 & 0,282 & 0,275 & 0,282 \\
2 & 0,275 & 0,274 & 0,282 & 0,286 \\
3 & 0,281 & 0,280 & 0,282 & 0,280 \\
4 & 0,281 & 0,285 & 0,282 & 0,275 \\
5 & 0,280 & 0,280 & 0,277 & 0,285 \\
\hline
\end{tabular}

Anova: fator único

\section{RESUMO}

\begin{tabular}{cclll}
\hline Grupo & Contagem & Soma & Média & Variância \\
\hline balde 1 & 5 & 1,4 & 0,28 & $9 \mathrm{E}-06$ \\
balde 2 & 5 & 1,401 & 0,2802 & $1,62 \mathrm{E}-05$ \\
balde 3 & 5 & 1,398 & 0,2796 & $1,13 \mathrm{E}-05$ \\
balde 4 & 5 & 1,408 & 0,2816 & $1,93 \mathrm{E}-05$ \\
\hline
\end{tabular}

\section{ANOVA}

\begin{tabular}{lcccccc}
\hline $\begin{array}{l}\text { Fonte } \\
\text { variação }\end{array}$ & $S Q$ & $g l$ & $M Q$ & $F$ & valor-P & $F$ crítico \\
\hline $\begin{array}{l}\text { Entre } \\
\text { grupos }\end{array}$ & $1,14 \mathrm{E}-05$ & 3 & $3,78 \mathrm{E}-06$ & 0,271207 & 0,845234 & 3,238867 \\
$\begin{array}{l}\text { Dentre } \\
\text { grupos }\end{array}$ & 0,000223 & 16 & $1,4 \mathrm{E}-05$ & & & \\
Total & 0,000235 & 19 & & & & \\
\hline
\end{tabular}

$\mathrm{u}_{\text {homogeneidade }}=\left(\mathrm{MQ}_{\text {dentre grupos }} / \mathrm{n}\right)^{1 / 2} \times\left[2 /\left(\mathrm{v}_{\mathrm{MQ} \text { dentre grupos }}\right)\right]^{1 / 4}$

Essa expressão é baseada na consideração de que se pode obter um intervalo de confiança para $s_{\text {entre grupos }}$. A meia amplitude do intervalo de confiança obtido com $95 \%$ de confiança, convertida

Tabela 3. Resultados da análise de variâncias - Determinação de cálcio

\begin{tabular}{ccccc}
\hline \% Ca & balde 1 & balde 2 & balde 3 & balde 4 \\
\hline 1 & 0,104 & 0,110 & 0,111 & 0,109 \\
2 & 0,106 & 0,109 & 0,104 & 0,106 \\
3 & 0,112 & 0,108 & 0,107 & 0,110 \\
4 & 0,109 & 0,105 & 0,109 & 0,109 \\
5 & 0,108 & 0,109 & 0,108 & 0,108 \\
\hline
\end{tabular}

Anova: fator único

RESUMO

\begin{tabular}{ccccc}
\hline Grupo & Contagem & Soma & Média & Variância \\
\hline balde 1 & 5 & 0,539 & 0,1078 & $9,2 \mathrm{E}-06$ \\
balde 2 & 5 & 0,541 & 0,1082 & $3,7 \mathrm{E}-06$ \\
balde 3 & 5 & 0,539 & 0,1078 & $6,7 \mathrm{E}-06$ \\
balde 4 & 5 & 0,542 & 0,1084 & $2,3 \mathrm{E}-06$ \\
\hline
\end{tabular}

ANOVA

\begin{tabular}{lcccccc}
\hline $\begin{array}{l}\text { Fonte } \\
\text { variação }\end{array}$ & $S Q$ & $g l$ & $M Q$ & $F$ & valor-P & $F$ crítico \\
\hline $\begin{array}{l}\text { Entre } \\
\text { grupos }\end{array}$ & $1,35 \mathrm{E}-06$ & 3 & $4,5 \mathrm{E}-07$ & 0,082192 & 0,968763 & 3,238867 \\
$\begin{array}{l}\text { Dentre } \\
\text { grupos }\end{array}$ & $8,76 \mathrm{E}-05$ & 16 & $5,47 \mathrm{E}-06$ & & & \\
Total & $8,89 \mathrm{E}-05$ & 19 & & & & \\
\hline
\end{tabular}

Tabela 4. Resultados da análise de variâncias - Determinação de alumínio

\begin{tabular}{ccccc}
\hline \%Al & balde 1 & balde 2 & balde 3 & balde 4 \\
\hline 1 & 0,083 & 0,090 & 0,089 & 0,088 \\
2 & 0,090 & 0,090 & 0,087 & 0,089 \\
3 & 0,089 & 0,092 & 0,088 & 0,084 \\
4 & 0,088 & 0,084 & 0,089 & 0,088 \\
5 & 0,088 & 0,085 & 0,089 & 0,088 \\
\hline
\end{tabular}

Anova: fator único

\section{RESUMO}

\begin{tabular}{cccll}
\hline Grupo & Contagem & Soma & Média & Variância \\
\hline balde 1 & 5 & 0,438 & 0,0876 & $7,3 \mathrm{E}-06$ \\
balde 2 & 5 & 0,441 & 0,0882 & $1,22 \mathrm{E}-05$ \\
balde 3 & 5 & 0,442 & 0,0884 & $8 \mathrm{E}-07$ \\
balde 4 & 5 & 0,437 & 0,0874 & $3,8 \mathrm{E}-06$ \\
\hline
\end{tabular}

\section{ANOVA}

\begin{tabular}{lcccccc}
\hline $\begin{array}{l}\text { Fonte } \\
\text { variação }\end{array}$ & $S Q$ & $g l$ & $M Q$ & $F$ & valor-P & $F$ crítico \\
\hline $\begin{array}{l}\text { Entre } \\
\text { grupos }\end{array}$ & $3,4 \mathrm{E}-06$ & 3 & $1,13 \mathrm{E}-06$ & 0,188105 & 0,902939 & 3,238867 \\
$\begin{array}{l}\text { Dentre } \\
\text { grupos }\end{array}$ & $9,64 \mathrm{E}-05$ & 16 & $6,02 \mathrm{E}-06$ & & & \\
Total & $9,98 \mathrm{E}-05$ & 19 & & & & \\
\hline
\end{tabular}


em incerteza padrão, pode ser considerada como a medida do impacto da repetitividade do método sobre a estimativa de $\mathrm{s}$

Usando a Equação 3 obtém-se $u_{\text {homogeneidade }}$ para cada um dos elementos considerados no estudo de homogeneidade. A Tabela 7

Tabela 5. Resultados da análise de variâncias - Determinação de manganês

\begin{tabular}{ccccc}
\hline$\%$ Mn & balde 1 & balde 2 & balde 3 & balde 4 \\
\hline 1 & 0,0120 & 0,0117 & 0,0120 & 0,0121 \\
2 & 0,0120 & 0,0120 & 0,0121 & 0,0120 \\
3 & 0,0113 & 0,0117 & 0,0117 & 0,0115 \\
4 & 0,0115 & 0,0120 & 0,0116 & 0,0117 \\
5 & 0,0116 & 0,0120 & 0,0119 & 0,0115 \\
\hline
\end{tabular}

Anova: fator único

\section{RESUMO}

\begin{tabular}{ccccl}
\hline Grupo & Contagem & Soma & Média & Variância \\
\hline balde 1 & 5 & 0,0584 & 0,01168 & $9,7 \mathrm{E}-08$ \\
balde 2 & 5 & 0,0594 & 0,01188 & $2,7 \mathrm{E}-08$ \\
balde 3 & 5 & 0,0593 & 0,01186 & $4,3 \mathrm{E}-08$ \\
balde 4 & 5 & 0,0588 & 0,01176 & $7,8 \mathrm{E}-08$ \\
\hline
\end{tabular}

\section{ANOVA}

\begin{tabular}{lcccccc}
\hline $\begin{array}{l}\text { Fonte } \\
\text { variação }\end{array}$ & $S Q$ & $g l$ & $M Q$ & $F$ & valor-P & $F$ crítico \\
\hline $\begin{array}{l}\text { Entre } \\
\text { grupos }\end{array}$ & $1,29 \mathrm{E}-07$ & 3 & $4,32 \mathrm{E}-08$ & 0,704762 & 0,562987 & 3,238867 \\
$\begin{array}{l}\text { Dentre } \\
\text { grupos }\end{array}$ & $9,8 \mathrm{E}-07$ & 16 & $6,13 \mathrm{E}-08$ & & & \\
Total & $1,11 \mathrm{E}-06$ & 19 & & & & \\
\hline
\end{tabular}

Tabela 6. Resultados da análise de variâncias - Determinação de titânio

\begin{tabular}{ccccc}
\hline$\%$ Ti & balde 1 & balde 2 & balde 3 & balde 4 \\
\hline 1 & 0,0103 & 0,0110 & 0,0108 & 0,0105 \\
2 & 0,0104 & 0,0106 & 0,0104 & 0,0106 \\
3 & 0,0109 & 0,0106 & 0,0109 & 0,0108 \\
4 & 0,0109 & 0,0102 & 0,0109 & 0,0105 \\
5 & 0,0106 & 0,0104 & 0,0105 & 0,0110 \\
\hline
\end{tabular}

Anova: fator único

\section{RESUMO}

\begin{tabular}{cccll}
\hline Grupo & Contagem & Soma & Média & Variância \\
\hline balde 1 & 5 & 0,0531 & 0,01062 & $7,7 \mathrm{E}-08$ \\
balde 2 & 5 & 0,0528 & 0,01056 & $8,8 \mathrm{E}-08$ \\
balde 3 & 5 & 0,0535 & 0,0107 & $5,5 \mathrm{E}-08$ \\
balde 4 & 5 & 0,0534 & 0,01068 & $4,7 \mathrm{E}-08$ \\
\hline
\end{tabular}

\section{ANOVA}

\begin{tabular}{lcccccc}
\hline $\begin{array}{l}\text { Fonte } \\
\text { variação }\end{array}$ & $S Q$ & $g l$ & $M Q$ & $F$ & valor-P & $F$ crítico \\
\hline $\begin{array}{l}\text { Entre } \\
\text { grupos }\end{array}$ & $6 \mathrm{E}-08$ & 3 & $2 \mathrm{E}-08$ & 0,299625 & 0,825174 & 3,238867 \\
$\begin{array}{l}\text { Dentre } \\
\text { grupos }\end{array}$ & $1,07 \mathrm{E}-06$ & 16 & $6,67 \mathrm{E}-08$ & & & \\
Total & $1,13 \mathrm{E}-06$ & 19 & & & & \\
\hline
\end{tabular}

mostra os valores para a variância devido à homogeneidade e que deverão ser adicionados à incerteza final do valor a ser certificado.

Tabela 7. Valores de variâncias devido à homogeneidade do material em lote candidato a material de referência para os elementos estudados

\begin{tabular}{cc}
\hline Elemento & Incerteza Padrão $\left(\mathrm{u}_{\text {homogeneidade }}\right)$ \\
\hline $\mathrm{Fe}$ & 0,0009948 \\
$\mathrm{Ca}$ & 0,0006219 \\
$\mathrm{Al}$ & 0,0006524 \\
$\mathrm{Mn}$ & 0,00006584 \\
$\mathrm{Ti}$ & 0,00006868 \\
$\mathrm{Cu}$ & 0,00001064 \\
\hline
\end{tabular}

Após realizado o teste de homogeneidade, foi conduzido um programa interlaboratorial para a etapa de certificação do lote preparado. Cerca de 12 laboratórios participaram desta etapa do trabalho. Nem todos determinaram todos os elementos solicitados (Tabela 8)

A avaliação estatística dos resultados encaminhados à coordenação do programa interlaboratorial foi feita usando a análise de variância fator único (laboratórios), após tratamento para os resultados "outliers"14. Cada laboratório recebeu dois frascos escolhidos aleatoriamente do lote preparado e realizou 3 replicatas independentes para cada frasco. As médias dos resultados e incertezas estão apresentados na Tabela 8.

Os valores para limites superior e inferior de cada elemento foram obtidos dos resultados do programa interlaboratorial. A incerteza indicada na Tabela 8 refere-se aos resultados das análises químicas efetuadas. Usando a Equação 4, a incerteza $u_{c}(x)$, associada ao valor a ser atribuído ao elemento a ser certificado, indica a incerteza que combina as duas variâncias: devido à caracterização e à homogeneidade.

$\mathrm{u}_{\mathrm{c}}(\mathrm{x})=\left(\mathrm{u}_{\text {caracterização }}^{2}+\mathrm{u}_{\text {homogeneidade }}^{2}\right)^{1 / 2}$

sendo $\mathrm{u}^{2}$ homogeneidade $=$ parcela calculada devido à homogeneidade; $\mathrm{u}_{\text {caracterização }}^{2}=(\mathrm{MQ} / \mathrm{pqn}) \mathrm{p}=\mathrm{n}^{\mathrm{o}}$ de laboratórios, $\mathrm{q}=\mathrm{n}^{\mathrm{o}}$ de frascos e $\mathrm{n}$ $=\mathrm{n}^{\mathrm{o}}$ de replicatas efetuadas por laboratório.

Usando a Equação 4, os valores para a incerteza padrão combinada, $\mathrm{u}_{\mathrm{c}}(\mathrm{x})$, para cada um dos elementos estudados estão mostrados na Tabela 9.

Nessa tabela, a incerteza expandida U(x) considera o fator de abrangência 1,96, para o grau de confiança de $95 \%$.

Expressando os resultados finais como valores de média e incerteza arredondados temos os seguintes limites: $(0,29-0,28) \% \mathrm{Fe}$; $(0,11-0,10) \% \mathrm{Ca} ;(0,087-0,082) \% \mathrm{Al} ;(0,012-0,011) \% \mathrm{Mn}$ e $(0,010-$ $0,0091) \%$ Ti.

Comparando esses limites com os obtidos na Tabela 8 de $(0,29$ $0,28) \% \mathrm{Fe} ;(0,11-0,10) \% \mathrm{Ca} ;(0,088-0,082) \% \mathrm{Al} ;(0,012-0,011) \%$ Mn e (0,011-0,0086)\% Ti , verifica-se que, para todos os elementos as incertezas dos respectivos valores médios se mantêm exceto para o elemento Ti, que teve sua amplitude aumentada de $(0,010$ $0,0091) \%$ para $(0,011-0,0086) \%$. Pode-se comentar que, ao se considerar a incerteza devido à não homogeneidade, os resultados finais não se mostraram diferentes, confirmando ser suficientemente homogêneo o lote de material a ser certificado.

\section{CONCLUSÃO}

Materiais de Referência são muito usados para dar validade a medições em química analítica e representam meios que relacio- 
Tabela 8. Média dos resultados obtidos por laboratórios participantes de programa interlaboratorial

\begin{tabular}{|c|c|c|c|c|c|}
\hline Elemento* & $\mathrm{Fe}$ & $\mathrm{Ca}$ & $\mathrm{Al}$ & $\mathrm{Mn}$ & $\mathrm{Ti}$ \\
\hline Média (X) & 0,2864 & 0,1030 & 0,0847 & 0,01143 & 0,00975 \\
\hline MQ & $8,55 \times 10^{-4}$ & $2,69 \times 10^{-4}$ & $1,16 \times 10^{-4}$ & $0,0512 \times 10^{-4}$ & $0,187 \times 10^{-4}$ \\
\hline $\mathrm{p}$ & 13 & 11 & 11 & 13 & 11 \\
\hline $\mathrm{q}$ & 2 & 2 & 2 & 2 & 2 \\
\hline $\mathrm{n}$ & 3 & 3 & 3 & 3 & 3 \\
\hline$T_{p-1}$ & 2,179 & 2,262 & 2,228 & 2,179 & 2,228 \\
\hline $\mathrm{u}_{\text {caracterizacão }}^{\mathrm{p}-1}$ & 0,0072 & 0,0045 & 0,0030 & 0,00056 & 0,0012 \\
\hline Limite Superior & 0,2936 & 0,1075 & 0,0877 & 0,0120 & 0,0110 \\
\hline Limite Inferior & 0,2792 & 0,0985 & 0,0817 & 0,0109 & 0,0086 \\
\hline
\end{tabular}

* resultados em \%; MQ: média quadrática; p: $\mathrm{n}^{\mathrm{o}}$ de laboratórios; q: $\mathrm{n}^{\mathrm{o}}$ de frascos; $\mathrm{n}: \mathrm{n}^{\mathrm{o}}$ de replicatas por frasco; $\mathrm{u}_{\text {caracterizaçã }}:$ incerteza da caracterização; $\mathrm{T}_{\mathrm{p}-1}$ - fator de probabilidade $95 \%$ confiança (distribuição t-Student)

Tabela 9. Resultados dos valores médios obtidos em programa interlaboratorial, com as incertezas combinada e expandida (devido à caracterização e a não homogeneidade do lote preparado)

\begin{tabular}{lccccc}
\hline Elemento & $\begin{array}{c}\text { Valor médio } \\
\mathrm{X}(\%)\end{array}$ & $\begin{array}{c}\text { Incerteza Padrão } \\
\text { Combinada } \mathrm{u}_{\mathrm{c}}(\mathrm{x})\end{array}$ & $\begin{array}{c}\text { Incerteza } \\
\text { Expandida*U(x) }\end{array}$ & $\begin{array}{c}\text { Limite } \\
\text { Superior(\%) }\end{array}$ & $\begin{array}{c}\text { Limite } \\
\text { Inferior(\%) }\end{array}$ \\
\hline $\mathrm{Fe}$ & 0,2864 & $2,521 \times 10^{-3}$ & $4,942 \times 10^{-3}$ & 0,2913 & 0,2815 \\
$\mathrm{Ca}$ & 0,1030 & $1,705 \times 10^{-3}$ & $3,343 \times 10^{-3}$ & 0,1063 & 0,0997 \\
$\mathrm{Al}$ & 0,0847 & $1,189 \times 10^{-3}$ & $2,330 \times 10^{-3}$ & 0,0870 & 0,0824 \\
$\mathrm{Mn}$ & 0,01143 & $0,191 \times 10^{-3}$ & $0,374 \times 10^{-3}$ & 0,0118 & 0,0111 \\
$\mathrm{Ti}$ & 0,00975 & $0,350 \times 10^{-3}$ & $0,687 \times 10^{-3}$ & 0,0104 & 0,0091 \\
\hline
\end{tabular}

*fator de abrangência $-\mathrm{t}$-Student $=1,96(95 \%$ de confiança $)$

nam os aspectos da qualidade de resultados analíticos com a rastreabilidade das medições obtidas. Através da informação sobre as incertezas associadas aos valores das propriedades certificadas, o laboratório usuário pode verificar se atinge ou não a exatidão e a precisão desejadas, ao introduzi-los nos processos analíticos a serem controlados.

No desenvolvimento de material candidato a material de referência, o teste de homogeneidade deve ser planejado para se poder conhecer as variabilidades devido à possível não homogeneidade do material embalado, a ser disponibilizado ao usuário. Ao se calcular a variância devido à homogeneidade, o produtor de material de referência tem meios de avaliar a qualidade do material que foi preparado e a ser disponibilizado.

Caso os aspectos de homogeneidade não sejam suficientes e ao não considerar a contribuição devida a esse fator, a qualidade do material de referência certificado, expressa pela sua incerteza, parecerá maior do que realmente é, pois não está sendo considerada a variância devido à não homogeneidade. Porém, conhecendo-se essa variância e ao considerá-la no cômputo da incerteza final do valor certificado, caso ela seja pequena, é evidenciada a real homogeneidade do material. Nesse caso a homogeneidade do material preparado é suficientemente boa para os propósitos desejados.

$\mathrm{O}$ teste de homogeneidade como uma das etapas importantes de um trabalho de desenvolvimento de material de referência não deve ser negligenciado. Apesar de trabalhoso, confere confiabilidade e qualidade ao material de referência.

\section{AGRADECIMENTOS}

À LIASA - Ligas de Alumínio S.A. pelo fornecimento do material bruto e à FAPESP pelo auxílio financeiro.

\section{REFERÊNCIAS}

1. De Bièvre, P.; Taylor, P. D. P.; Metrologia 1997, 34, 67.

2. VIM - Vocabulário Internacional de Termos Fundamentais e Gerais de Metrologia, INMETRO/SENAI, 2e ed., Brasília, 2000.

3. Pauwels, J.; Van der Veen, A. M. H.; Lamberty, A.; Schimmel, H.; Accreditation and Quality Assurance 2000, 5, 95.

4. Valcárcel, M.; Principles of Analytical Chemistry. A Textbook, SpringerVerlag: Berlin, 2000, p. 101.

5. Associação Brasileira de Normas Técnicas - NBR/ISO GUIA 30: Termos e Definições Relacionados com Materiais de Referência, Rio de Janeiro, 2001.

6. Associação Brasileira de Normas Técnicas - NBR/ISO GUIA 32: Calibração em Química Analítica e Uso de Materiais de Referência Certificados, Rio de Janeiro, 2001.

7. Meyer, K.; Matschat, R. Em Reference Materials in Analytical Chemistry; Zschunke, A.; Zunger, A.; Hull, R.; Osgood Jr., R. M.; Sakaki, H., eds.; Springer - Verlag Berlin Heidelberg New York, 2000, p. 58.

8. International Organization for Standardization - ISO Guide 35: Certification of reference materials - General and Statistical Principles, Switzerland, 1989

9. International Organization for Standardization - ISO GUM: Guia para Expressão da Incerteza de Medição, $2^{\mathrm{a}}$ ed. brasileira e revisada, Agosto 1998.

10. Ilnat, M. Em Reference Materials for Chemical Analysis: Certification, Availability and Proper Usage; Stoeppler, M.; Wolf, W. R.; Jenks, P. J., eds.; Wiley-VCH Verlag: Germany, 2001, p. 40.

11. Associação Brasileira de Normas Técnicas - NBR/ISO/IEC 17025: Requisitos Técnicos para a Competência Técnica de Laboratórios de Ensaios e Calibração, Rio de Janeiro, 2001.

12. Linsinger, T.; Pauwels, J.; Van der Veen, A. M. H.; Schimmel, H.; Lamberty, A.; Accreditation and Quality Assurance 2001, 6, 20.

13. Van der Veen, A. M. H.; Linsinger, T.; Pauwels, J.; Accreditation and Quality Assurance 2001, 6, 26.

14. International Organization for Standardization - ISO/DIS 5725 -Accuracy (trueness and precision) of Measurement Methods and Results. Part 2: A basic method for the determination of repeatability and reproducibility of a standard measurement method, Geneva: Switzerland, 1990. 